\title{
PURE TORSION OF COMPRESSIBLE NONLINEARLY ELASTIC CIRCULAR CYLINDERS
}

\author{
BY \\ DEBRA A. POLIGNONE AND CORNELIUS O. HORGAN \\ University of Virginia, Charlottesville, Virginia
}

\begin{abstract}
The large deformation torsion problem of an elastic circular cylinder, composed of homogeneous isotropic compressible nonlinearly elastic material and subjected to twisting moments at its ends, is described. The problem is formulated as a two-point boundary-value problem for a second-order nonlinear ordinary differential equation in the radial deformation field. The class of materials for which pure torsion (i.e., a deformation with zero radial displacement) is possible is described. Specific material models are used to illustrate the results.
\end{abstract}

Introduction. The finite torsion of an elastic circular cylinder due to applied twisting moments at its ends was one of the classic problems solved by Rivlin [1,2] for isotropic incompressible nonlinearly elastic materials. The nonhomogeneous deformation found by Rivlin is sustainable, in the absence of body force, in all homogeneous isotropic incompressible materials. Thus it is a universal, or controllable, deformation. Furthermore, by virtue of the constraint of zero volume change, the deformation is that of pure torsion so that there is no extension in the radial direction and the cross-section of the cylinder remains circular.

The situation for compressible materials is considerably more complicated. First, by virtue of Ericksen's theorem [3], the deformation cannot be universal. Second, there will, in general, be some radial extension. The torsion problem for a circular cylinder composed of a homogeneous isotropic compressible nonlinearly elastic material has been formulated by Green [4] (see also Green and Adkins [5, p. 67]) and properties of the solution discussed. The formulation leads to a nonlinear secondorder ordinary differential equation for the radial deformation $r(R)$ (see Eq. (2.22) of the present paper). Here $R$ and $r$ denote radial components of a cylindrical polar coordinate system in the undeformed and deformed configurations respectively. The deformation $r(R)$ depends on the form of the strain-energy density. An equivalent version of the ordinary differential equation may be written in terms of the principal stretches [6] (see Eq. (7.8) of the present paper). As remarked in [4, 6], an analytic solution of the governing equation, even for very special strain-energy functions, is not readily obtained and so numerical approaches are called for. Other aspects of

Received August 7, 1990.

(C)1991 Brown University 
the compressible torsion problem which have been investigated include second-order effects (see, e.g., [7-9]) and the influence of small compressibility (see, e.g., [10-12] and the references cited therein).

In this paper, we are concerned with a special issue, namely that of examining the class of compressible materials for which pure torsion is possible. This question was first raised by Currie and Hayes [13] in their study of nonuniversal deformations. In the next section, we briefly summarize the relevant equations of finite elastostatics leading to the governing ordinary differential equation (2.22) and associated boundary conditions. In Sec. 3, we consider the special case of the Blatz-Ko material [14] for which pure torsion, in the absence of lateral surface tractions, is possible. This was recently observed by Beatty in [15], and independently by Carroll and Horgan in [16]. The stress distribution in torsion for the Blatz-Ko material is remarkably simple-simpler even than the corresponding stress field for a neo-Hookean incompressible material. In Sec. 4, we establish a necessary condition on the strain-energy density of the material for pure torsion to be possible by seeking solutions of the form $r=R$ of the governing ordinary differential equation. In Sec. 5, a subclass of materials for which pure torsion is possible is identified. This includes the Hadamard material discussed in [13] and a family of Blatz-Ko materials previously investigated in $[15,16]$. In Sec. 6, a wider range of materials is examined. In the final section, an alternative criterion in terms of the principal stretches is described, and some examples involving specific material models are discussed.

Issues similar to those addressed here have been examined recently by the present authors [28] for finite axial shear deformations of compressible nonlinearly elastic hollow cylinders. The inner surface of the tube is bonded to a rigid cylinder while the outer surface is subjected to a uniformly distributed axial shear traction and the radial traction is zero. The class of compressible materials for which axisymmetric antiplane shear (i.e., a deformation with zero radial displacement and axial displacement depending only on the radial coordinate) is possible is identified in [28]. The results in [28] and the present paper complement one another in the sense that many of the materials described in [28] for which finite axisymmetric anti-plane shear is possible do not sustain pure torsion while, conversely, several of the admissible materials identified here do not sustain axisymmetric anti-plane shear.

2. Formulation of torsion problem. We are concerned with torsional deformation of an elastic solid circular cylinder due to applied twisting moments at its ends. Thus the deformation, which takes the point with cylindrical polar coordinates $(R, \Theta, Z)$ in the undeformed region to the point $(r, \theta, z)$ in the deformed region, has the form

$$
r=r(R), \quad \theta=\Theta+\tau Z, \quad z=Z,
$$

with $\dot{r} \equiv d r / d R>0$, where the constant $\tau>0$ is the twist per unit undeformed length. For a cylinder composed of an incompressible isotropic elastic material, the classic solution of Rivlin [1] shows that pure torsional deformations, that is, deformations of the form (2.1) with

$$
r=R,
$$


are possible. Thus the deformed configuration is again a solid circular cylinder which undergoes no volume change. For a compressible material, pure torsion will not be possible in general. The radial deformation $r(R)$ is governed by a second-order nonlinear ordinary differential equation (see Eq. (2.22) below).

The strain-energy density per unit undeformed volume for isotropic elastic compressible materials is given by

$$
W=W\left(I_{1}, I_{2}, I_{3}\right),
$$

where $I_{1}, I_{2}, I_{3}$ are the principal invariants of the deformation tensor $\mathbf{B}=\mathbf{F F}^{\mathrm{T}}$, where $\mathbf{F}$ is the deformation gradient tensor. We have

$$
\begin{aligned}
& I_{1}=\operatorname{tr} \mathbf{B}=\lambda_{1}^{2}+\lambda_{2}^{2}+\lambda_{3}^{2}, \\
& I_{2}=\operatorname{tr} \mathbf{B}^{*}=\lambda_{1}^{2} \lambda_{2}^{2}+\lambda_{2}^{2} \lambda_{3}^{2}+\lambda_{3}^{2} \lambda_{1}^{2}, \\
& I_{3}=\operatorname{det} \mathbf{B}=\lambda_{1}^{2} \lambda_{2}^{2} \lambda_{3}^{2},
\end{aligned}
$$

where $\mathbf{B}^{*}=(\operatorname{det} \mathbf{B}) \mathbf{B}^{-1}$ is the adjoint of $\mathbf{B}$ and $\lambda_{i}(i=1,2,3)$ are the principal stretches. The corresponding response equation for the Cauchy stress tensor $\mathbf{T}$ is (see, e.g., [8])

$$
\mathbf{T}=\beta_{0} \mathbf{1}+\beta_{1} \mathbf{B}+\beta_{-1} \mathbf{B}^{-1},
$$

where 1 is the unit tensor. Here the elastic response functions $\beta_{s}=\beta_{s}\left(I_{1}, I_{2}, I_{3}\right)$ $(s=0,1,-1)$ are given by

$$
\beta_{0}=2 I_{3}^{-1 / 2}\left[I_{2} W_{2}+I_{3} W_{3}\right], \quad \beta_{1}=2 I_{3}^{-1 / 2} W_{1}, \quad \beta_{-1}=-2 I_{3}^{1 / 2} W_{2},
$$

where $W_{i}=\partial W / \partial I_{i}(i=1,2,3)$. We shall assume that the strain-energy $W$ and the stress $\mathbf{T}$ vanish in the undeformed state where $I_{1}=3, I_{2}=3, I_{3}=1$, and so

$$
W(3,3,1)=0, \quad W_{1}(3,3,1)+2 W_{2}(3,3,1)+W_{3}(3,3,1)=0 .
$$

Corresponding to the deformation field (2.1), we have

$$
\begin{gathered}
\mathbf{F}=\left(\begin{array}{ccc}
\dot{r} & 0 & 0 \\
0 & r / R & \tau r \\
0 & 0 & 1
\end{array}\right), \\
\mathbf{B}=\left(\begin{array}{ccc}
\dot{r}^{2} & 0 & 0 \\
0 & r^{2} / R^{2}+\tau^{2} r^{2} & \tau r \\
0 & \tau r & 1
\end{array}\right), \\
\mathbf{B}^{-1}=\left(\begin{array}{ccc}
\dot{r}^{-2} & 0 & 0 \\
0 & R^{2} / r^{2} & -\tau R^{2} / r \\
0 & -\tau R^{2} / r & 1+\tau^{2} R^{2}
\end{array}\right), \\
I_{1}=1+\dot{r}^{2}+\frac{r^{2}}{R^{2}}+\tau^{2} r^{2}, \\
I_{2}=\frac{r^{2}}{R^{2}}+\dot{r}^{2}+\frac{\dot{r}^{2} r^{2}}{R^{2}}+\tau^{2} \dot{r}^{2} r^{2},
\end{gathered}
$$




$$
I_{3}=\frac{\dot{r}^{2} r^{2}}{R^{2}} .
$$

Substitution from (2.9), (2.10) in (2.5) yields the physical components of the Cauchy stress $\mathbf{T}$ as

$$
\begin{gathered}
T_{r r}=\beta_{0}+\beta_{1} \dot{r}^{2}+\beta_{-1} \dot{r}^{-2}, \\
T_{\theta \theta}=\beta_{0}+\beta_{1}\left(\frac{r^{2}}{R^{2}}+\tau^{2} r^{2}\right)+\beta_{-1} \frac{R^{2}}{r^{2}}, \\
T_{z z}=\beta_{0}+\beta_{1}+\beta_{-1}\left(1+\tau^{2} R^{2}\right), \\
T_{\theta z}=\beta_{1}(\tau r)-\frac{\tau R^{2}}{r} \beta_{-1}, \\
T_{r \theta}=0, \quad T_{r z}=0,
\end{gathered}
$$

where the $\beta_{s}(s=0,1,-1)$ given by (2.6) are evaluated at the values of the invariants given by $(2.11)-(2.13)$. Note that, since $r=r(R)$, it is convenient for us to consider $\mathbf{T}=\mathbf{T}(R)$ rather than the more conventional $\mathbf{T}=\mathbf{T}(r)$.

The equilibrium equations, in the absence of body forces are

$$
\operatorname{div} \mathbf{T}=\mathbf{0},
$$

which in the present case, reduce to the single equation

$$
\frac{\partial T_{r r}}{\partial r}+\frac{1}{r}\left(T_{r r}-T_{\theta \theta}\right)=0 .
$$

Using the chain rule, we write $(2.20)$ as

$$
\frac{\partial T_{r r}}{\partial R}+\frac{\dot{r}}{r}\left(T_{r r}-T_{\theta \theta}\right)=0 .
$$

On using (2.14), (2.15), (2.6), we find that (2.21) can be written as

$$
\begin{aligned}
& \frac{d}{d R}\left[\frac{R \dot{r}}{r} W_{1}+\frac{r \dot{r}}{R} W_{3}+\left(\frac{R \dot{r}}{r}+\frac{r \dot{r}}{R}+\tau^{2} R \dot{r} r\right) W_{2}\right] \\
& \quad+W_{1}\left(\frac{R \dot{r}^{2}}{r^{2}}-\frac{1}{R}-\tau^{2} R\right)+W_{2}\left(\frac{R \dot{r}^{2}}{r^{2}}-\frac{1}{R}\right)=0,
\end{aligned}
$$

where the $W_{i}(i=1,2,3)$ are evaluated at the values $(2.11)-(2.13)$ for the invariants. Since $r=r(R)$, we have used the ordinary derivative symbol in (2.22). Equation (2.22) is a (highly) nonlinear second-order ordinary differential equation for the function $r(R)$. This equation may be obtained from analysis presented in Green [4] (see also Green and Adkins [5, p. 67]). Following developments presented by Ogden [6, pp. 260-263], an equivalent equation, involving the principal stretches, may also be obtained (see Eq. (7.8) of the present paper). As remarked by these authors, the task of obtaining analytical solutions to (2.22) (or its counterpart (7.8)), even for simple forms of the strain-energy, is formidable.

For a solid circular cylinder of undeformed radius $A$ subjected to end torques only, the boundary conditions of traction-free lateral surface will be satisfied if

$$
T_{r r}(A)=0,
$$


since $T_{r \theta}=T_{r z} \equiv 0$ by (2.18). In addition, to ensure that $\mathbf{F}$ is bounded, we impose the regularity condition

$$
r(R)=O(R) \quad \text { as } R \rightarrow 0 .
$$

Thus the two-point boundary value problem which arises is to solve (2.22) for $r(R)$ on $0<R<A$ subject to the conditions (2.23), (2.24).

3. The Blatz-Ko material. It has been shown recently by Beatty [15] and independently by Carroll and Horgan [16] that pure torsion is possible for a special compressible material proposed by Blatz and Ko [14] as a model for foam rubber material. In this section, we examine this special case in detail.

The strain-energy density function describing the Blatz-Ko material is given by

$$
W=\frac{\mu}{2}\left(\frac{I_{2}}{I_{3}}+2 I_{3}^{1 / 2}-5\right)
$$

( $\mu=$ constant is the shear modulus for infinitesimal deformations; we take $\mu>0$ ), for which the stress response equation takes the simple form

$$
\mathbf{T}=\mu\left(\mathbf{1}-I_{3}^{-1 / 2} \mathbf{B}^{-1}\right) .
$$

It is easily verified that (2.7) is satisfied. The response of the material (3.1) for a variety of stress and strain states has been examined by Knowles and Sternberg [17]. Beatty [15] has provided an extensive discussion of the material (3.1) as well as its generalized form as proposed by Blatz and Ko [14]. The experimental basis for (3.1), as advocated by Blatz and Ko [14], is described in [15,17]. It should be noted that the response of the material (3.1), even to infinitesimal deformations, is special with value $\frac{1}{4}$ for Poisson's ratio. Nevertheless, the material model (3.1) has recently attracted considerable attention. In particular, the analytic solution of a wide variety of problems in finite elastostatics for compressible materials has been possible for this material (see $[15,16,18-21])$.

When $W$ is given by (3.1), the differential equation $(2.22)$ for $r(R)$ reduces to

$$
3 R r^{3} \ddot{r}-r^{3} \dot{r}+R^{3}(\dot{r})^{4}=0,
$$

where $\dot{r}=d r / d R$ and $\ddot{r}=d^{2} r / d R^{2}$. We note that the twist per unit undeformed length $\tau$ does not appear in (3.3). The second-order nonlinear ordinary differential equation (3.3) is identical to the equilibrium equation, derived in [18], governing plane strain axisymmetric deformations of the Blatz-Ko material (3.1); see Eq. (2.9) of [18]. This was also observed in [16]. The stresses $(2.14)-(2.18)$, for the material (3.1), are

$$
\begin{gathered}
T_{r r}=\mu\left(1-\frac{R}{r \dot{r}^{3}}\right), \quad T_{\theta \theta}=\mu\left(1-\frac{R^{3}}{r^{3} \dot{r}}\right), \\
T_{z z}=\mu\left[1-\frac{\left(1+\tau^{2} R^{2}\right) R}{r \dot{r}}\right], \quad T_{\theta z}=\mu \frac{\tau R^{3}}{r^{2} \dot{r}}, \\
T_{r \theta}=0, \quad T_{r z}=0 .
\end{gathered}
$$

Observe that the stresses (3.4) are independent of $\tau$. In fact, these stresses are also identical to the corresponding stresses in the plane strain axisymmetric problem (see 
Eq. (2.7) of [18]). In the latter problem, $T_{r r}$ and $T_{\theta \theta}$ are the only nonzero stresses [18].

Solutions of Eq. (3.3) have been discussed extensively in [18-21]. For our purposes here, it is sufficient to note that $r(R)=R$ is a solution of (3.3). An equivalent observation was also made in [16]. The regularity condition (2.24) is clearly satisfied. When $r=R$, Eq. (3.4) shows that

$$
T_{r r} \equiv 0, \quad T_{\theta \theta} \equiv 0,
$$

and so the boundary condition (2.23) is satisfied. Thus the Blatz-Ko material (3.1) can sustain pure torsion with traction-free lateral surface (cf. $[15,16])$. As remarked in [16], this is true for both a solid and hollow cylinder. From (3.5) we find that the only nonzero stresses are

$$
T_{\theta z}=\mu \tau R, \quad T_{z z}=-\mu \tau^{2} R^{2} .
$$

The stress distribution given by (3.6)-(3.8) is remarkably simple. This stress state is even simpler than the stress state arising in pure torsion of a neo-Hookean incompressible material where the normal stresses $T_{r r}$ and $T_{\theta \theta}$ are not zero. From (3.7), (3.8) we see that

$$
T_{\theta \theta}-T_{z z}=\tau R T_{z \theta}
$$

holds, a universal relation for all Blatz-Ko materials (3.1). This was also established in [15]. From (3.8), we see that the axial normal stress $T_{z z}$ is compressive. The resultant axial compressive force $T$ and twisting moment $M$ are defined by

$$
T=\int_{D} T_{z z} d A, \quad M=\int_{D} R T_{\theta z} d A,
$$

where $D$ denotes the cross-section of the cylinder. From (3.8), (3.10) one finds that

$$
T=-\mu \tau^{2} \pi \frac{A^{4}}{2}, \quad M=\mu \tau \pi \frac{A^{4}}{2}
$$

so that

$$
-T=\tau M .
$$

This relationship was also obtained in [15] and independently in [16]. As pointed out by Beatty [15], (3.12) is a universal relation between applied twisting moment and compressive thrust, valid for all Blatz-Ko materials of the form (3.1).

4. Pure torsion: a necessary condition. With the results of the preceding section as motivation, we proceed to examine the class of compressible materials which can sustain pure torsion.

A necessary condition on $W$ for pure torsion to be possible follows immediately on setting $r=R$ in (2.22). From (2.11)-(2.13), we find that, for pure torsional deformations,

$$
I_{1}=I_{2}=3+\tau^{2} R^{2}, \quad I_{3}=1,
$$

and so the deformation is isochoric. On setting $r=R$ in (2.22), we obtain

$$
\frac{d}{d R}\left[W_{1}+W_{3}+\left(2+\tau^{2} R^{2}\right) W_{2}\right]-\tau^{2} R W_{1}=0 .
$$


On employing the chain rule, (4.2) may be written as

$$
2\left(3+\tau^{2} R^{2}\right) \widehat{W}_{21}+2\left(2+\tau^{2} R^{2}\right) \widehat{W}_{22}+2\left(\widehat{W}_{31}+\widehat{W}_{32}+\widehat{W}_{11}\right)+2 \widehat{W}_{2}-\widehat{W}_{1}=0,
$$

where $W_{i j}=\partial^{2} W / \partial I_{i} \partial I_{j}$, and the superposed hat notation is used to indicate that the corresponding quantities are evaluated at the values (4.1) for the invariants $I_{i}(i=$ $1,2,3)$. The condition (4.3) is a necessary condition on $W$ for pure torsion to be possible. The implications of (4.3) will be our concern in the next two sections of this paper. A condition equivalent to (4.2) was obtained by Currie and Hayes [13, Eq. (5.5)].

When $r=R$, the corresponding stresses follow from (2.14)-(2.18) as

$$
\begin{gathered}
T_{r r}=\widehat{\beta}_{0}+\widehat{\beta}_{1}+\widehat{\beta}_{-1}, \\
T_{\theta \theta}=\widehat{\beta}_{0}+\widehat{\beta}_{1}\left(1+\tau^{2} R^{2}\right)+\widehat{\beta}_{-1}, \\
T_{z z}=\widehat{\beta}_{0}+\widehat{\beta}_{1}+\widehat{\beta}_{-1}\left(1+\tau^{2} R^{2}\right), \\
T_{\theta z}=\tau R\left(\widehat{\beta}_{1}-\widehat{\beta}_{-1}\right), \\
T_{r \theta}=0, \quad T_{r z}=0,
\end{gathered}
$$

where $\widehat{\beta}_{s}=\beta_{s}\left(3+\tau^{2} R^{2}, 3+\tau^{2} R^{2}, 1\right)$ are given by (2.6). For any $W$ for which (4.3) holds, the boundary condition (2.23) will be satisfied if

$$
\widehat{\beta}_{0}+\widehat{\beta}_{1}+\widehat{\beta}_{-1}=0 \text { on } R=A,
$$

where we recall that $A$ is the radius of the solid cylinder. We note from (4.5), (4.6) that for all compressible materials for which (4.3) is satisfied, the universal relation

$$
T_{\theta \theta}-T_{z z}=\tau R T_{z \theta}
$$

holds. This is, of course, the analog of the well-known universal relation for torsion of incompressible materials.

5. A subclass of materials. We begin our analysis of (4.3) by considering materials for which the dependence of $W$ on $I_{1}$ and $I_{2}$ is linear, so that $\widehat{W}_{21}=0, \widehat{W}_{22}=0$, $\widehat{W}_{11}=0$ in (4.3). Thus, in particular, the terms in (4.3) which depend explicitly on the nonconstant quantity $\tau^{2} R^{2}$ will vanish identically. We write $W$ as

$$
W=\frac{\mu}{2}\left[H_{1}\left(I_{3}\right)\left(I_{1}-3\right)+H_{2}\left(I_{3}\right)\left(I_{2}-3\right)+H_{3}\left(I_{3}\right)\right]
$$

where the constant $\mu>0$ and the functions $H_{i}\left(I_{3}\right)(i=1,2,3)$ are assumed to satisfy

$$
H_{3}(1)=0, \quad H_{1}(1)+2 H_{2}(1)+H_{3}^{\prime}(1)=0,
$$

so that $(2.7)$ holds. The prime notation denotes differentiation with respect to argument. On substitution from (5.1) in (4.3) and evaluating at the values (4.1), we find that

$$
2 H_{1}^{\prime}(1)-H_{1}(1)+2 H_{2}^{\prime}(1)+2 H_{2}(1)=0 .
$$

Thus, (5.3) is a necessary condition on $H_{\alpha}\left(I_{3}\right)(\alpha=1,2)$ for pure torsion to be possible for the class of materials (5.1). 
We consider some examples of materials of the form (5.1). First, it is seen that the Blatz-Ko material (3.1) can be written in the form (5.1) if we choose

$$
H_{1}\left(I_{3}\right)=0, \quad H_{2}\left(I_{3}\right)=I_{3}^{-1}, \quad H_{3}\left(I_{3}\right)=3 I_{3}^{-1}+2 I_{3}^{1 / 2}-5 .
$$

It is easily checked that (5.2) holds. Since $H_{1}\left(I_{3}\right)=0$, condition (5.3) reduces to

$$
2 \mathrm{H}_{2}^{\prime}(1)+2 \mathrm{H}_{2}(1)=0
$$

which is seen to be satisfied by the choice of $\mathrm{H}_{2}\left(I_{3}\right)$ given in (5.4). Thus we recover the result, established in a different way in Sec. 3, that $r=R$ is a solution of the differential equation (2.22) for the Blatz-Ko material. As we saw in Sec. 3, the boundary condition (2.23) is also satisfied in this case.

The Blatz-Ko material (3.1) is a special case of a more general material model also developed by Blatz and Ko in [14]. This generalized Blatz-Ko material is given by

$$
\begin{aligned}
W= & \frac{\mu}{2} f\left\{I_{1}-1-\frac{1}{\nu}+\frac{(1-2 \nu)}{\nu} I_{3}^{-\nu /(1-2 \nu)}\right\} \\
& +\frac{\mu}{2}(1-f)\left\{\frac{I_{2}}{I_{3}}-1-\frac{1}{\nu}+\frac{(1-2 \nu)}{\nu} I_{3}^{\nu /(1-2 \nu)}\right\},
\end{aligned}
$$

where the constants $\mu, \nu$, and $f$ are such that $\mu>0,0 \leq f \leq 1$, and $0<\nu<\frac{1}{2}$. The constants $\mu$ and $\nu$ are the shear modulus and Poisson's ratio for infinitesimal deformations while $f$ measures the volume fraction of voids in the foam-rubber material modeled by (5.6). On setting $f=0, \nu=\frac{1}{4}$ in (5.6) one recovers (3.1). The material (5.6) can be expressed in the form (5.1) if one takes

$$
\begin{aligned}
& H_{1}\left(I_{3}\right)=f, \quad H_{2}\left(I_{3}\right)=(1-f) I_{3}^{-1}, \\
H_{3}\left(I_{3}\right)= & f\left[-\frac{1}{\nu}+2+\frac{(1-2 \nu)}{\nu} I_{3}^{-\nu /(1-2 \nu)}\right] \\
& +(1-f)\left[3 I_{3}^{-1}-1-\frac{1}{\nu}+\frac{(1-2 \nu)}{\nu} I_{3}^{\nu /(1-2 \nu)}\right] .
\end{aligned}
$$

It is readily verified that the normalization conditions (5.2) are satisfied. The necessary condition (5.3) for pure torsion is seen to be satisfied by (5.7) if and only if

$$
f=0 .
$$

This result was also obtained by Beatty (see [15, p. 1723]). Thus, for the material

$$
W=\frac{\mu}{2}\left\{\frac{I_{2}}{I_{3}}-1-\frac{1}{\nu}+\frac{(1-2 \nu)}{\nu} I_{3}^{\nu /(1-2 \nu)}\right\}
$$

the equilibrium equations (2.22) are satisfied by $r=R$. The corresponding stresses may be found from (4.4)-(4.8), (2.6). It turns out that they are identical to the stresses found in Sec. 3 for the Blatz-Ko material (3.1). In particular, the tractionfree boundary condition (2.23) is satisfied and so materials described by (5.10) can sustain pure torsional deformations with traction free lateral surface.

We next consider the Hadamard material

$$
W=c_{1}\left(I_{1}-3\right)+c_{2}\left(I_{2}-3\right)+H\left(I_{3}\right),
$$


where $c_{1}, c_{2}$ are constants and $H\left(I_{3}\right)$ is, as yet, an arbitrary function. This is of the form $(5.1)$ if we choose

$$
H_{1}\left(I_{3}\right)=\frac{2 c_{1}}{\mu}, \quad H_{2}\left(I_{3}\right)=\frac{2 c_{2}}{\mu}, \quad H_{3}\left(I_{3}\right)=2 \frac{H\left(I_{3}\right)}{\mu} .
$$

To ensure that the normalization conditions (5.2) hold, we require

$$
H(1)=0, \quad H^{\prime}(1)+c_{1}+2 c_{2}=0 .
$$

With $H_{i}\left(I_{3}\right)$ given by $(5.12)$, it is seen that $(5.3)$ holds if

$$
2 c_{2}=c_{1} \text {. }
$$

Thus the differential equation for pure torsion is satisfied for the Hadamard material (5.11) provided (5.14) holds. This result was obtained in a different way in [13].

The stresses (4.4)-(4.8) for the Hadamard material (5.11) are

$$
\begin{gathered}
T_{r r}=2 c_{2} \tau^{2} R^{2}, \quad T_{\theta \theta}=2\left(c_{1}+c_{2}\right) \tau^{2} R^{2}, \\
T_{z z}=0, \quad T_{\theta z}=2\left(c_{1}+c_{2}\right) \tau R, \\
T_{r \theta}=0, \quad T_{r z}=0 .
\end{gathered}
$$

Thus, even when (5.14) is imposed, the boundary condition (2.23) of traction-free lateral surface cannot be satisfied except in the degenerate case where $c_{2}=0$. This may be contrasted with the results for the Blatz-Ko and generalized Blatz-Ko materials with $f=0$. Observe also from (5.16) that the axial stress $T_{z z}$ vanishes identically for the Hadamard material. Thus to sustain a pure torsional deformation of a Hadamard material satisfying (5.14), a uniformly distributed tensile loading $\left(c_{2}>0\right)$ would be required on the lateral surface of the cylinder.

To conclude this section, we return to the basic necessary condition for pure torsion (4.3) and observe that the only derivatives of $W_{3}$ appearing in (4.3) are $\widehat{W}_{31}$ and $\widehat{W}_{32}$. Thus a single term in $W$ which is a function of $I_{3}$ only automatically satisfies (4.3). Furthermore, the function $\mathrm{H}_{3}\left(I_{3}\right)$ in (5.1) does not enter in the criterion (5.3). We also note that any $W$ of the form

$$
W=k\left(I_{1}-3\right)+H\left(I_{3}\right) \quad(k \text { constant })
$$

(so that $H_{2}=0$ in $(5.1)$ and $H_{1}\left(I_{3}\right)$ is constant) cannot satisfy (5.3) unless $k=0$. Thus pure torsion cannot be sustained by materials of the form (5.18) with $k \neq 0$. A variety of constitutive laws of the form (5.18) have been proposed in the literature.

6. A more general class of materials. The task of seeking a representation for the most general form of $W$ for which (4.3) is satisfied is formidable. Here we shall pursue a more modest objective, namely that of broadening the subclass of materials discussed in Sec. 5.

Rather than seeking $W$ for which $\widehat{W}_{\alpha \beta}=0(\alpha, \beta=1,2)$, we merely require that

$$
\widehat{W}_{21}+\widehat{W}_{22}=0 \text {. }
$$


This will again ensure, as in Sec. 5, that the terms in (4.3) which depend explicitly on $\tau^{2} R^{2}$ will vanish identically. Any function $W\left(I_{1}, I_{2}, I_{3}\right)$ of the form $W=$ $P\left(I_{1}-I_{2}, I_{3}\right)$, where $P$ is a sufficiently smooth function, satisfies

$$
W_{21}+W_{22}=0 \text {, }
$$

so that, in particular, (6.1) holds for such $W$. With this motivation, we thus consider the class of materials for which $W$ can be written as

$$
\begin{aligned}
W=\frac{\mu}{2}\left[P\left(I_{1}-I_{2}, I_{3}\right)+Q\left(I_{1},\right.\right. & \left.I_{3}\right)+R\left(I_{2}\right) S\left(I_{3}\right) \\
& \left.+H_{1}\left(I_{3}\right)\left(I_{1}-3\right)+H_{2}\left(I_{3}\right)\left(I_{2}-3\right)+H_{3}\left(I_{3}\right)\right],
\end{aligned}
$$

where $\mu>0$, and $P, Q, R, S, H_{i}(i=1,2,3)$ are sufficiently smooth functions of the arguments indicated in (6.3). The functions $H_{i}$ are not the same as those of (5.1), except of course when $P, Q, R, S$ are identically zero. It is readily verified that $W$ as given in (6.3) satisfies (6.2) (and so (6.1)) if and only if $R\left(I_{2}\right)$ is a linear function and so

$$
R\left(I_{2}\right)=k_{1} I_{2}+k_{2},
$$

where $k_{1}, k_{2}$ are arbitrary constants. On redefining $S\left(I_{3}\right), H_{3}\left(I_{3}\right)$ to include these constants, we rewrite $(6.3)$ as

$$
\begin{aligned}
W=\frac{\mu}{2}\left[P\left(I_{1}-I_{2}, I_{3}\right)+Q\left(I_{1},\right.\right. & \left.I_{3}\right)+I_{2} S\left(I_{3}\right) \\
& \left.+H_{1}\left(I_{3}\right)\left(I_{1}-3\right)+H_{2}\left(I_{3}\right)\left(I_{2}-3\right)+H_{3}\left(I_{3}\right)\right],
\end{aligned}
$$

which thus satisfies (6.1). The normalization conditions $(2.7)$ will be satisfied by (6.5) if

$$
P(0,1)+Q(3,1)+3 S(1)+H_{3}(1)=0
$$

and

$$
\begin{aligned}
& -P_{1}(0,1)+P_{2}(0,1)+\frac{\partial Q}{\partial I_{1}}(3,1)+\frac{\partial Q}{\partial I_{3}}(3,1) \\
& +3 S^{\prime}(1)+2 S(1)+H_{1}(1)+2 H_{2}(1)+H_{3}^{\prime}(1)=0
\end{aligned}
$$

where the notation

$$
P_{1}(x, y)=\frac{\partial}{\partial x} P(x, y), \quad P_{2}(x, y)=\frac{\partial}{\partial y} P(x, y)
$$

is used to denote the derivative of $P\left(I_{1}-I_{2}, I_{3}\right)$ with respect to its arguments. When $P, Q, S$ are set equal to zero in (6.6), (6.7), one recovers (5.2).

Using (6.1), we see that condition (4.3) reduces to

$$
2\left(\widehat{W}_{21}+\widehat{W}_{31}+\widehat{W}_{32}+\widehat{W}_{11}\right)+2 \widehat{W}_{2}-\widehat{W}_{1}=0 \text {. }
$$

Our task now is to find a condition on the functions $P, Q, S, H_{i}$ appearing in (6.5) which will ensure that (6.9) holds. When $P, Q, S$ are set equal to zero, this should reduce to (5.3). On substitution from (6.5) into (6.9), one finds that

$$
\begin{aligned}
-3 & P_{1}(0,1)+\left(2 \frac{\partial^{2} Q}{\partial I_{1}^{2}}+2 \frac{\partial^{2} Q}{\partial I_{1} \partial I_{3}}-\frac{\partial Q}{\partial I_{1}}\right)_{I_{1}=3+\tau^{2} R^{2}, I_{3}=1} \\
& +2\left[S^{\prime}(1)+S(1)\right]+2 H_{1}^{\prime}(1)-H_{1}(1)+2 H_{2}^{\prime}(1)+2 H_{2}(1)=0 .
\end{aligned}
$$


When $P, Q, S$ are set equal to zero, we see that (6.10) does indeed reduce to (5.3).

We shall not attempt to describe the most general class of functions $P, Q, S, H_{i}$ $(i=1,2,3)$ for which $(6.10)$ holds, subject to (6.6), (6.7), but rather indicate some of the possibilities. The form of (6.10) suggests that one might seek $Q\left(I_{1}, I_{3}\right)$ such that, for all $I_{1} \geq 3, I_{3} \geq 1$,

$$
2 \frac{\partial^{2} Q}{\partial I_{1}^{2}}+2 \frac{\partial^{2} Q}{\partial I_{1} \partial I_{3}}-\frac{\partial Q}{\partial I_{1}}=0
$$

A separation of variables in (6.11) leads to a representation for $Q$ of the form

$$
Q\left(I_{1}, I_{3}\right)=\alpha e^{\beta\left(I_{3}-1\right)} e^{(1 / 2-\beta)\left(I_{1}-3\right)}
$$

to within an arbitrary additive function of $I_{3}$. Without loss of generality, this additive function may be included in $H_{3}\left(I_{3}\right)$ in the representation (6.5). In (6.12), $\alpha$ and $\beta \neq \frac{1}{2}$ are constants. It may be readily verified that (6.12) satisfies (6.11). Thus the sum of the three terms in (6.10) involving $Q$ are zero, when $Q$ is given by (6.12).

We remark that (6.12) is just one possible solution of (6.11). In fact, (6.11) has an immediate first integral and the resulting first-order partial differential equation can be shown to have a solution of the form

$$
Q\left(I_{1}, I_{3}\right)=e^{\left(I_{3}-1\right) / 2}\left[F\left(I_{1}-I_{3}\right)+\int_{0}^{I_{3}-1} G(s) e^{-s / 2} d s\right],
$$

where $F$ and $G$ are sufficiently smooth. When $G=0$ and $F=\alpha \exp \left[\left(\frac{1}{2}-\beta\right)\left(I_{1}-\right.\right.$ $\left.I_{3}-2\right)$ ] in $(6.12)^{\prime}$, one recovers $(6.12)$. For simplicity in what follows, we use the form (6.12) for $Q$.

With regard to the function $S\left(I_{3}\right)$, one might seek $S$ such that

$$
S^{\prime}(1)+S(1)=0 \text {, }
$$

so that (6.10) is further simplified. One choice which satisfies (6.13) is $S\left(I_{3}\right)=k / I_{3}$, where $k$ is a constant. This choice for $S$ in (6.5) yields a term $k I_{2} / I_{3}$ which was encountered in the Blatz-Ko and generalized Blatz-Ko materials (3.1) and (5.10) respectively. Another possibility is to choose $S$ such that

$$
S^{\prime}\left(I_{3}\right)+S\left(I_{3}\right)=0 \text { for all } I_{3} \geq 1
$$

so that

$$
S\left(I_{3}\right)=k e^{-I_{3}}=\gamma e^{-\left(I_{3}-1\right)}, \quad \gamma, k \text { constants. }
$$

This yields a term of the form

$$
I_{2} S\left(I_{3}\right)=\gamma I_{2} e^{-\left(I_{3}-1\right)}
$$

in the strain-energy $W$ in (6.5). Strain-energies with exponential terms of the form $(6.12)^{\prime},(6.15)$ have been successfully used in biomechanical modeling (see, e.g., [15, $22,23]$ and the references cited therein).

Suppose then that $Q, S$ are chosen as in (6.12), (6.15). The condition (6.10) now reads

$$
-3 P_{1}(0,1)+2 H_{1}^{\prime}(1)-H_{1}(1)+2 H_{2}^{\prime}(1)+2 H_{2}(1)=0,
$$


while the normalization conditions $(6.6),(6.7)$ reduce to

$$
\begin{gathered}
P(0,1)+\alpha+3 \gamma+H_{3}(1)=0, \\
-P_{1}(0,1)+P_{2}(0,1)+\frac{\alpha}{2}-\gamma+H_{1}(1)+2 H_{2}(1)+H_{3}^{\prime}(1)=0 .
\end{gathered}
$$

Thus, a material described by a strain-energy function $W$ of the form (6.5), with $Q, S$ chosen as in (6.12), (6.15), can sustain pure torsional deformations provided (6.17) holds.

As an example, suppose we augment the Hadamard material (5.11) by adding to the $W$ of (5.11) the terms $Q$ and $S$, given by (6.12) and (6.15), so that

$$
W=\frac{\mu}{2}\left[Q\left(I_{1}, I_{3}\right)+I_{2} S\left(I_{3}\right)+c_{1}\left(I_{1}-3\right)+c_{2}\left(I_{2}-3\right)+H\left(I_{3}\right)\right]
$$

where, for convenience, the multiplicative factor $\mu / 2$ has been introduced in the portion of $W$ describing a Hadamard material. On comparing (6.20) with (6.5), we thus have

$$
P=0, \quad H_{1}\left(I_{3}\right)=c_{1}, \quad H_{2}\left(I_{3}\right)=c_{2}, \quad H_{3}\left(I_{3}\right)=H\left(I_{3}\right) .
$$

The normalization conditions (6.18), (6.19) now read

$$
\begin{gathered}
\alpha+3 \gamma+H(1)=0, \\
\alpha / 2-\gamma+c_{1}+2 c_{2}+H^{\prime}(1)=0 .
\end{gathered}
$$

With $P, H_{i}(i=1,2,3)$ given by $(6.21)$, it is seen that $(6.17)$ holds if

$$
2 c_{2}=c_{1} \text {. }
$$

Thus the differential equation for pure torsion is satisfied for the material (6.20), where $Q, S$ are as in (6.12), (6.15), provided (6.24) holds. This is a generalization of the result obtained in Sec. 5 for Hadamard materials.

The radial stress for the material (6.20) can be found using (4.4), (2.6) and so

$$
2 T_{r r} / \mu=\tau^{2} R^{2} c_{1}+\alpha e^{(1 / 2-\beta) \tau^{2} R^{2}}-\alpha,
$$

where $(6.23),(6.24)$ have also been employed. Thus the traction-free boundary condition (2.23) at the lateral surface $R=A$ of the cylinder would be satisfied if the constants $\alpha, \beta, c_{1}$ were related by

$$
\tau^{2} A^{2} c_{1}+\alpha e^{(1 / 2-\beta) \tau^{2} A^{2}}-\alpha=0
$$

Another interesting special class of materials is found from (6.5) on taking $P=0$, $Q=0, H_{\alpha}\left(I_{3}\right)=0(\alpha=1,2)$. The necessary condition (6.10) for pure torsion then reduces to

$$
S^{\prime}(1)+S(1)=0
$$

while the normalization conditions $(6.6),(6.7)$ reduce to

$$
3 S(1)+H_{3}(1)=0, \quad 3 S^{\prime}(1)+2 S(1)+H_{3}^{\prime}(1)=0 .
$$

When (6.27) holds, (6.28) can be combined to yield

$$
S(1)=H_{3}^{\prime}(1), \quad 3 H_{3}^{\prime}(1)+H_{3}(1)=0 .
$$


Thus any material of the form

$$
W=\frac{\mu}{2}\left[I_{2} S\left(I_{3}\right)+H_{3}\left(I_{3}\right)\right],
$$

will sustain pure torsion provided $(6.27),(6.29)$ hold. It is readily verified that for the material (6.30), subject to (6.27) and (6.29), $T_{r r}=0$ and so the traction-free boundary condition (2.23) is satisfied. Moreover, the remaining stresses are

$$
\begin{gathered}
T_{\theta z}=\mu S(1) \tau R, \quad T_{z z}=-\mu S(1) \tau^{2} R^{2}, \\
T_{\theta \theta}=0, \quad T_{r \theta}=0, \quad T_{r z}=0 .
\end{gathered}
$$

The Blatz-Ko materials (3.1), (5.10) are seen to be special cases of (6.30).

7. A necessary condition in terms of the principal stretches. Up to now we have considered isotropic compressible elastic materials with strain-energy density $W$ expressed as a function of the principal invariants $I_{1}, I_{2}, I_{3}$. In this section, we briefly outline how the preceding considerations are modified when $W$ is expressed directly as a function of the principal stretches $\lambda_{1}, \lambda_{2}, \lambda_{3}$ or, equivalently, as a function of the principal invariants $i_{1}, i_{2}, i_{3}$ of the stretch tensor, where

$$
i_{1}=\lambda_{1}+\lambda_{2}+\lambda_{3}, \quad i_{2}=\lambda_{1} \lambda_{2}+\lambda_{2} \lambda_{3}+\lambda_{3} \lambda_{1}, \quad i_{3}=\lambda_{1} \lambda_{2} \lambda_{3} .
$$

Such alternative formulations have been widely investigated particularly for compressible materials (see, e.g., $[6,24]$ and the references cited therein).

When

$$
W=w\left(i_{1}, i_{2}, i_{3}\right),
$$

it has been shown by Carroll [24] that the analog of (2.5) is

$$
\mathbf{T}=\gamma_{0} \mathbf{1}+\gamma_{1} \mathbf{v}+\gamma_{-1} \mathbf{v}^{-1}
$$

where $\mathbf{V}$ is the left stretch tensor in the polar decomposition for $\mathbf{F}=\mathbf{V R}$. In (7.3), the elastic response functions $\gamma_{s}=\gamma_{s}\left(i_{1}, i_{2}, i_{3}\right)(s=0,1,-1)$ are given by

$$
\gamma_{0}=i_{3}^{-1}\left(i_{2} \frac{\partial w}{\partial i_{2}}+i_{3} \frac{\partial w}{\partial i_{3}}\right), \quad \gamma_{1}=i_{3}^{-1} \frac{\partial w}{\partial i_{1}}, \quad \gamma_{-1}=-\frac{\partial w}{\partial i_{2}} .
$$

The normalization conditions analogous to (2.7) are then

$$
w(3,3,1)=0, \quad \frac{\partial w}{\partial i_{1}}+2 \frac{\partial w}{\partial i_{2}}+\left.\frac{\partial w}{\partial i_{3}}\right|_{i_{1}=i_{2}=3, i_{3}=1}=0 .
$$

For the deformation (2.1), the principal stretches are given by

$$
\lambda_{1}=\dot{r}, \quad \lambda_{2}^{2}+\lambda_{3}^{2}=\frac{r^{2}}{R^{2}}+\tau^{2} r^{2}+1, \quad \lambda_{2} \lambda_{3}=\frac{r}{R} .
$$

Using the notation

$$
w\left(i_{1}, i_{2}, i_{3}\right)=W^{*}\left(\lambda_{1}, \lambda_{2}, \lambda_{3}\right),
$$

the equilibrium equations (2.19) can be shown (see, e.g., [6, p. 263]) to reduce to the single equation

$$
\frac{d}{d R}\left(R W_{1}^{*}\right)=\frac{1}{\lambda_{2} \lambda_{3}}\left[\frac{\lambda_{2}^{3} W_{2}^{*}-\lambda_{3}^{3} W_{3}^{*}-\lambda_{2} W_{2}^{*}+\lambda_{3} W_{3}^{*}}{\lambda_{2}^{2}-\lambda_{3}^{2}}\right],
$$


where $W_{i}^{*} \equiv \partial W^{*} / \partial \lambda_{i}(i=1,2,3)$ are evaluated at the values given by (7.6) for the principal stretches. Equation (7.8), which is equivalent to (2.22), is again a secondorder nonlinear ordinary differential equation for $r(R)$. The boundary conditon (2.23), when expressed in the present notation (cf. [6, p. 263]), reads

$$
W_{1}^{*}(A)=0 .
$$

Thus the two-point boundary value problem which arises is to solve (7.8) for $r(R)$ on $0<R<A$ subject to the boundary condition (7.9) and the regularity condition (2.24).

Pure torsion. On setting $r=R$ in (7.8), we obtain a necessary condition on $W^{*}$ for pure torsion to be possible. When $r=R$, we find from (7.6) that

$$
\lambda_{1}=1, \quad \lambda_{2}^{2}+\lambda_{3}^{2}=2+\tau^{2} R^{2}, \quad \lambda_{2} \lambda_{3}=1,
$$

and (7.8) yields

$$
\left(\lambda_{2}^{2}-\lambda_{3}^{2}\right) \frac{d}{d R}\left(R W_{1}^{*}\right)=\lambda_{2}^{3} W_{2}^{*}-\lambda_{3}^{3} W_{3}^{*}-\lambda_{2} W_{2}^{*}+\lambda_{3} W_{3}^{*} .
$$

On employing the chain rule, (7.11) may be written as

$$
\begin{gathered}
\left(\lambda_{2}^{2}-1\right)\left(1-\lambda_{3}^{2}\right)\left(\lambda_{2} \widehat{W}_{12}^{*}-\lambda_{3} \widehat{W}_{13}^{*}\right)+\left(\lambda_{2}^{2}-\lambda_{3}^{2}\right) \widehat{W}_{1}^{*} \\
-\lambda_{2}\left(\lambda_{2}^{2}-1\right) \widehat{W}_{2}^{*}+\lambda_{3}\left(\lambda_{3}^{2}-1\right) \widehat{W}_{3}^{*}=0,
\end{gathered}
$$

where $W_{i j}^{*}=\partial^{2} W / \partial \lambda_{i} \partial \lambda_{j}$ and the superposed hat notation indicates that the corresponding quantities are evaluated at the values (7.10) for the principal stretches. The necessary condition (7.12) is the counterpart of (4.3).

Subclasses of materials. In what follows, we consider three classes of materials of the form (7.2) for which a wide range of deformations have been examined by Carroll [24]. We adopt the terminology of [24].

Class I. Here

$$
w=f\left(i_{1}\right)+c_{2}\left(i_{2}-3\right)+c_{3}\left(i_{3}-1\right),
$$

where $c_{2}, c_{3}$ are constants. Using a prime to denote differentiation with respect to argument, we assume that

$$
f(3)=0, \quad f^{\prime}(3)=-\left(2 c_{2}+c_{3}\right),
$$

so that (7.5) are satisfied. These are the harmonic materials introduced by John [25]. Corresponding to (7.13), in the notation of (7.7), we have

$$
\begin{aligned}
W^{*}\left(\lambda_{1}, \lambda_{2}, \lambda_{3}\right)= & f\left(\lambda_{1}+\lambda_{2}+\lambda_{3}\right)+c_{2}\left(\lambda_{1} \lambda_{2}+\lambda_{2} \lambda_{3}+\lambda_{3} \lambda_{1}-3\right) \\
& +c_{3}\left(\lambda_{1} \lambda_{2} \lambda_{3}-1\right) .
\end{aligned}
$$

On substitution from (7.15) into (7.12), one finds that the necessary condition for pure torsion reads

$$
\tau^{2} R^{2} f^{\prime \prime}+\left(f^{\prime}-c_{2}\right)\left[\lambda_{2}+\lambda_{3}-\left(2+\tau^{2} R^{2}\right)\right]=0,
$$

where the $\lambda_{i}(i=1,2,3)$ are given by (7.10). Clearly (7.16) will not be satisfied, in general, since $f$ is an arbitrary function subject only to the normalization conditions (7.14). 
Consider the special case when

$$
f^{\prime \prime} \equiv 0
$$

Then (7.16) will be satisfied if

$$
f^{\prime}(s)=c_{2},
$$

where $s=1+\lambda_{2}+\lambda_{3}$ and $\lambda_{2}, \lambda_{3}$ are related through (7.10). If one makes the stronger assumption that

$$
f^{\prime}(x) \equiv c_{2} \text { for all } x \geq 1,
$$

which is consistent with (7.17), then on integration of (7.19) and using the first condition in (7.14), we get

$$
f\left(i_{1}\right)=c_{2} i_{1}-3 c_{2} .
$$

However, the second condition in (7.14) will be satisfied only if

$$
c_{3}=-3 c_{2} \text {. }
$$

Thus, in view of $(7.21),(7.20)$, the strain-energy (7.13) reduces to the special purely linear form

$$
w=c_{2}\left(i_{1}-3\right)+c_{2}\left(i_{2}-3\right)-3 c_{2}\left(i_{3}-1\right) .
$$

As pointed out by Carroll [24, p. 71], the response in infinitesimal deformations of a material described by (7.22) is unrealistic physically.

Thus, we conclude that, except in the special case (7.22), the harmonic material described by (7.13) does not sustain pure torsional deformations.

Class II. Here

$$
w=c_{1}\left(i_{1}-3\right)+g\left(i_{2}\right)+c_{3}\left(i_{3}-1\right),
$$

where $c_{1}, c_{3}$ are constants and

$$
g(3)=0, \quad g^{\prime}(3)=-\frac{1}{2}\left(c_{1}+c_{3}\right),
$$

so that (7.5) are satisfied. We have

$$
\begin{aligned}
W^{*}= & c_{1}\left(\lambda_{1}+\lambda_{2}+\lambda_{3}-3\right)+g\left(\lambda_{1} \lambda_{2}+\lambda_{2} \lambda_{3}+\lambda_{3} \lambda_{1}\right) \\
& +c_{3}\left(\lambda_{1} \lambda_{2} \lambda_{3}-1\right) .
\end{aligned}
$$

On substitution from (7.25) into (7.12), the necessary condition for pure torsion reads

$$
\left(\lambda_{2}+\lambda_{3}\right) \tau^{2} R^{2} g^{\prime \prime}+\left(c_{1}-g^{\prime}\right)\left[\lambda_{2}+\lambda_{3}-\left(2+\tau^{2} R^{2}\right)\right]=0 .
$$

On comparing (7.26) with (7.16), it is seen that both conditions are of similar structure. Using arguments analogous to those employed for Class I, it can again be shown that, except for the special (nonphysical) case of a purely linear material $((7.22)$ with $c_{2}$ replaced by $c_{1}$ ), materials of Class II do not sustain pure torsional deformations. Class III. Here

$$
w=c_{1}\left(i_{1}-3\right)+c_{2}\left(i_{2}-3\right)+h\left(i_{3}\right),
$$

where $c_{1}, c_{2}$ are constants and

$$
h(1)=0, \quad h^{\prime}(1)=-\left(c_{1}+2 c_{2}\right),
$$


so that (7.5) are satisfied. The materials described by (7.27), with $c_{2}=0$, have been called Varga materials [26], since they may be viewed as a generalization, to include the effect of compressibility, of an incompressible material model proposed by Varga [27]. Corresponding to (7.27), we have

$$
W^{*}=c_{1}\left(\lambda_{1}+\lambda_{2}+\lambda_{3}-3\right)+c_{2}\left(\lambda_{1} \lambda_{2}+\lambda_{2} \lambda_{3}+\lambda_{3} \lambda_{1}-3\right)+h\left(\lambda_{1} \lambda_{2} \lambda_{3}\right) .
$$

On substitution from (7.29) into (7.12), one obtains

$$
\left(c_{1}-c_{2}\right)\left[\lambda_{2}+\lambda_{3}-\left(2+\tau^{2} R^{2}\right)\right]=0
$$

We observe that the constitutive function $h$ does not appear in (7.30), in contrast to the situation encountered in (7.16), (7.26) for materials of Class I, II respectively. The second term in (7.30) is easily seen to be nonzero for $\tau>0$ and so we conclude from (7.30) that materials of Class III can sustain pure torsion if

$$
c_{1}=c_{2} \text {. }
$$

This is analogous to the result for Hadamard materials discussed in Sec. 5.

It remains to examine the possibility of satisfying the traction-free boundary condition (7.9). When $W^{*}$ is given by (7.29), Eq. (7.9) reads

$$
c_{1}+c_{2}\left(\lambda_{2}+\lambda_{3}\right)+h^{\prime}(1)=0 \text { at } R=A,
$$

which can be written as

$$
c_{1}\left(1+\lambda_{2}+\lambda_{3}\right)+h^{\prime}(1)=0 \text { at } R=A
$$

on using (7.31). This condition is incompatible with the second condition in (7.28) and (7.31) since $\lambda_{2}+\left.\lambda_{3}\right|_{R=A} \neq 2$, as follows from (7.10) for $\tau>0$. Thus, similar to the situation encountered for Hadamard materials in Sec. 5, a uniformly distributed tensile loading would be required on the lateral surface of the cylinder in order for a Class III material satisfying (7.31) to sustain pure torsion.

Acknowledgments. This research was supported in part by the National Science Foundation under Grant No. MSM-89-04719 and by the U.S. Air Force Office of Scientific Research under Grant No. AFOSR-89-0470. The research of D. A. Polignone was also supported by a National Defense Science and Engineering Graduate Fellowship (NDSEGF) awarded by the U. S. Air Force. We are grateful to A. S. Wineman for helpful discussions which stimulated our interest in this problem.

\section{REFERENCES}

[1] R. S. Rivlin, Large elastic deformations of isotropic materials IV. Further developments of the general theory, Philos. Trans. Roy. Soc. London Ser. A 241, 379-397 (1948)

[2] R. S. Rivlin, A note on the torsion of an incompressible highly-elastic cylinder, Proc. Cambridge Philos. Soc. 45, 485-487 (1949)

[3] J. L. Ericksen, Deformations possible in every compressible, isotropic, perfectly elastic material, J. Math. Phys. 34, 126-128 (1955)

[4] A. E. Green, Finite elastic deformation of compressible isotropic bodies, Proc. Roy. Soc. London Ser. A 227, 271-278 (1955)

[5] A. E. Green and J. E. Adkins, Large Elastic Deformations, Oxford Univ. Press, Oxford, 1960

[6] R. W. Ogden, Non-linear Elastic Deformations, Ellis Horwood, Chichester, 1984 
[7] A. E. Green and E. W. Wilkes, $A$ note on the finite extension and torsion of a circular cylinder of compressible elastic isotropic material, Quart. J. Mech. Appl. Math. 6, 240-249 (1953)

[8] C. Truesdell and W. Noll, The non-linear field theories of mechanics, Handbuch der Physik, vol. III/3 (S. Flügge, ed.), Springer, Berlin, 1965

[9] R. T. Shield, An energy method for certain second-order effects with application to torsion of elastic bars under tension, J. Appl. Mech. 47, 75-81 (1980)

[10] M. Levinson, Finite torsion of slightly compressible rubberlike circular cylinders, Internat. J. NonLinear Mech. 7, 445-463 (1972)

[11] M. G. Faulkner and J. B. Haddow, Nearly isochoric finite torsion of a compressible isotropic elastic circular cylinder, Acta Mech. 13, 245-253 (1972)

[12] R. W. Ogden, Nearly isochoric elastic deformations: application to rubberlike solids, J. Mech. Phys. Solids 26, 37-57 (1978)

[13] P. K. Currie and M. Hayes, On non-universal finite elastic deformations, Finite Elasticity (D. E. Carlson and R. T. Shield, eds.), Proceedings of IUTAM Symposium, Martinus Nijhoff, The Hague, 1982, pp. 143-150

[14] P. J. Blatz and W. L. Ko, Application of finite elasticity to the deformation of rubbery materials, Trans. Soc. Rheol. 6, 223-251 (1962)

[15] M. F. Beatty, Topics in finite elasticity: hyperelasticity of rubber, elastomers, and biological tissueswith examples, Appl. Mech. Rev. 40, 1699-1734 (1987)

[16] M. M. Carroll and C. O. Horgan, Finite strain solutions for a compressible elastic solid, Quart. Appl. Math. 48, 767-780 (1990)

[17] J. K. Knowles and E. Sternberg, On the ellipticity of the equations of nonlinear elastostatics for a special material, J. Elasticity 5, 341-361 (1975)

[18] R. Abeyaratne and C. O. Horgan, Initiation of localized plane deformations at a circular cavity in an infinite compressible nonlinearly elastic medium, J. Elasticity 15, 243-256 (1985)

[19] C. O. Horgan and R. Abeyaratne, A bifurcation problem for a compressible nonlinearly elastic medium: growth of a micro-void, J. Elasticity 16, 189-200 (1986)

[20] D.-T. Chung, C. O. Horgan, and R. Abeyaratne, The finite deformation of internally pressurized hollow cylinders and spheres for a class of compressible elastic materials, Internat. J. Solids and Structures 22, 1557-1570 (1986)

[21] C. O. Horgan, Some remarks on axisymmetric solutions in finite elastostatics for compressible materials, Proc. Roy. Irish Acad. Sect. A 89, 185-193 (1989)

[22] J. S. K. Wong and R. T. Shield, The stability of a cylindrical elastic membrane of biological tissue and the effect of internal fluid flow, Ing.-Archiv 49, 393-412 (1980)

[23] Y. C. Fung, Biomechanics. Mechanical Properties of Living Tissues, Springer-Verlag, Berlin, 1981

[24] M. M. Carroll, Finite strain solutions in compressible isotropic elasticity, J. Elasticity 20, 65-92 (1988)

[25] F. John, Plane elastic waves of finite amplitude: Hadamard materials and harmonic materials, Comm. Pure Appl. Math. 19, 309-341 (1966)

[26] D. M. Haughton, Inflation of thick-walled compressible elastic spherical shells, IMA J. Appl. Math. 39, 259-272 (1987)

[27] O. H. Varga, Stress-Strain Behavior of Elastic Materials, Wiley, New York, 1966

[28] D. A. Polignone and C. O. Horgan, Axisymmetric finite anti-plane shear of compressible nonlinearly elastic circular tubes, Quart. Appl. Math., to appear 